\title{
Hybrid control scheme for distributed energy resource management in a market context
}

Han, Xue; Bindner, Henrik W.; Mehmedalic, Jasmin; Tackie, David Victor

Published in:

Proceedings of 2015 IEEE PES General Meeting, Conference \& Exposition

Link to article, DOI:

10.1109/PESGM.2015.7286000

Publication date:

2015

Document Version

Peer reviewed version

Link back to DTU Orbit

Citation (APA):

Han, X., Bindner, H. W., Mehmedalic, J., \& Tackie, D. V. (2015). Hybrid control scheme for distributed energy resource management in a market context. In Proceedings of 2015 IEEE PES General Meeting, Conference \& Exposition IEEE. https://doi.org/10.1109/PESGM.2015.7286000

\section{General rights}

Copyright and moral rights for the publications made accessible in the public portal are retained by the authors and/or other copyright owners and it is a condition of accessing publications that users recognise and abide by the legal requirements associated with these rights.

- Users may download and print one copy of any publication from the public portal for the purpose of private study or research.

- You may not further distribute the material or use it for any profit-making activity or commercial gain

- You may freely distribute the URL identifying the publication in the public portal 


\section{Hybrid control scheme for distributed energy resource management in a market context}

\author{
Xue HAN and Henrik W. BINDNER \\ Technical University of Denmark \\ Frederiskborgvej 399, Roskilde, Denmark \\ Email: xueh, hwbi@elektro.dtu.dk
}

\author{
Jasmin MEHMEDALIC and David V. TACKIE \\ Danish Energy Association \\ Rosenørns Allé 9, Frederiksberg, Denmark \\ Email: jme,dvt @ danskenergi.dk
}

\begin{abstract}
In modernizing the electricity grid, distributed energy resources (DERs) can play an important role in accommodating intermittent energy sources, assisting system operation and the transition to a smart grid. Proper aggregation and coordination of the available DER units is required to provide flexibility to meet regular demand from the distribution system operator (DSO). By considering both their physical constraints and the economical system operation, this paper proposes a realtime hybrid management system for DER units in a market environment, which considers both the request from the DSO and their local conditions. Voltage regulation service is formulated and implemented in the control frame. The performance is evaluated through simulation on an existing Danish MV and LV distribution grid and is compared with a local control method and the passive operation condition.
\end{abstract}

Index Terms-Distributed energy resource (DER), distribution system management, hybrid control frame, voltage control

\section{INTRODUCTION}

The goal of energy system sustainability is driving a booming growth of distributed renewable sources (e.g., wind and photovoltaic (PV)) in the power system [1]. The substitution of fossil fuels for electricity leads to increasing numbers of electric vehicles and heat pumps connected to the existing distribution system [1]. Distributed energy resources (DERs) are defined as small units that are installed at the end-users in the distribution grid and are able to adjust their production or consumption if demanded by external instances. The adjustable portion is referred as flexibility. Compared to the conventional regulating facilities in the system, DERs could be an attractive alternative candidate to provide system services, e.g., voltage regulation. Many voltage control solutions are proposed in the literature to mitigate negative impacts of DER integration and existing grid-operating problems [2], [3]. However, there lacks some considerations related to their deployment, especially in a liberal market environment [4].

In iPower, a Danish research and innovation project, a FLExibility Clearing House (FLECH) is proposed to enable transparent trading of flexibility and grid support services, especially on the distribution grid operation [5]. In this context, the hierarchical control scheme presented in [6] is further developed to be compatible with FLECH. The roles in the hierarchical control setup are distinguished by the physical

This work is supported by Danish national project iPower - a Strategic Platform for Innovation and Research within Intelligent Electricity (SPIR). structure of the power system, and modular functionalities are associated with the roles in different levels of the hierarchy. The hierarchy is built in a flexible and fault-tolerant manner where the roles in different levels of the hierarchy are elected from the controllers of available physical units [7].

Under the frame of FLECH, the responsibility and information exchange between different roles in the hierarchy is further clarified. The capability of the control scheme is also extended to multiple voltage levels (i.e., medium voltage (MV) and low voltage (LV) levels). A hybrid control scheme is proposed in this work, which contains both a hierarchical control frame for the flexibility aggregation and dispatch, and autonomous controllers at the bottom to accommodate both the local grid and request from the upper grid that provide robustness to the entire design. The novel control setup provides a good coordination of goals from different participants in a market context.

The remaining part of the paper is structured as follows: Section II proposes the tailored control scheme in a market environment, and discusses how the control method could assist grid operation, and Section III describes the detailed algorithm that is implemented in the control scheme to enable the functionality for service execution. It is followed by the case study in Section IV, which contains the simulation results and analysis. The paper is concluded in Section V.

\section{Hierarchical AgGregation Structure}

DER units have the capability to curtail or stimulate their power consumption or production, both active and reactive power, i.e., flexibility. Aggregating large amount and different types of units is essential for obtaining a system level scale.

\section{A. Proposed voltage control structure}

In [6], a hierarchical aggregation and control structure is proposed (see Fig. 1). The aggregation is based on the physical features of DER units, i.e., location, and other properties. Subsequently, DERs are dynamically grouped under a local controller (LC), which handles the optimal dispatch of the local resources. Furthermore, the present LCs are aggregated into the second level by the supervisory controller (SC). The aggregation also follows the natural topology of power distribution grid. The flexibility from different DER units are interfaced by the unit controllers (UC) to LCs. 


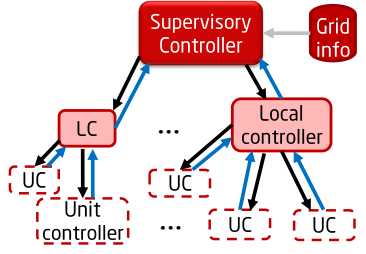

(a) Control hierarchy

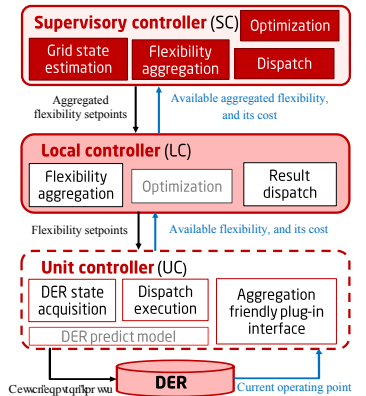

(b) Roles
Fig. 1. Sketch map of the hierarchical controller.

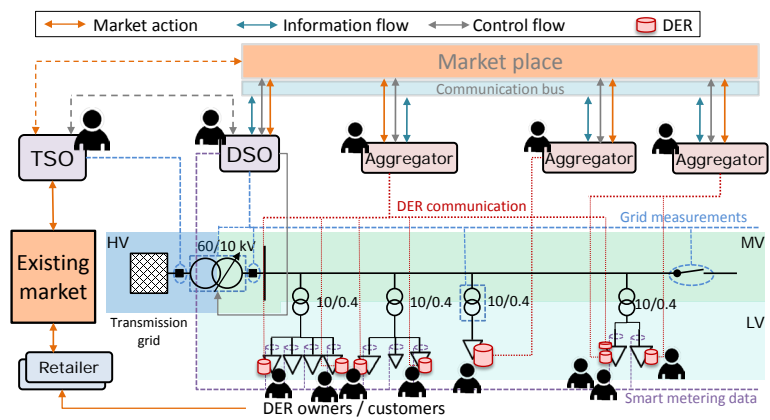

Fig. 2. Market based distribution grid operation with DER integration.

\section{B. Extended structure in FLECH context}

The provision of flexibility from DERs to the system involves several stakeholders. Fig. 2 presents the interactions among the stakeholders and their responsibilities. In the figure, FLECH stands as the new market place integrated with the necessary communication structure. It allows and enables efficient and transparent information exchange between different stakeholders. The distribution system operator (DSO) owns the grid assets and has the responsibility to maintain their operation. The data from the grid and from smart meters is collected and maintained by the DSO. Based on the current grid state, the DSO could make control decisions for all the controllable devices, such as switches and OLTCs. Unlike grid facilities, DER units are owned by customers. Therefore, the DSO can hardly access to the complete information and full controllability of the DER units. Meanwhile, the aggregator, who aggregates and coordinates the behaviour of the DER units has no access to observe the grid status and operate the grid. Naturally, the responsibilities of the grid and the portfolio of DER units are separated between the DSO and the aggregator. However, from Fig. 1, we see that the SC is in charge of both the decision for DER units and also the grid operation. Hence, the proposed hierarchical control structure in Fig. 1 can hardly be realized under such context.

Based on the responsibility and ownership of resources, a tailored control scheme is proposed as shown in Fig. 3. The functionality of SC is separated and allocated to different stakeholders. The map between the geographical location and electrical locations of certain DER units is used to translate the information between the DSO and aggregators. The DSO uses

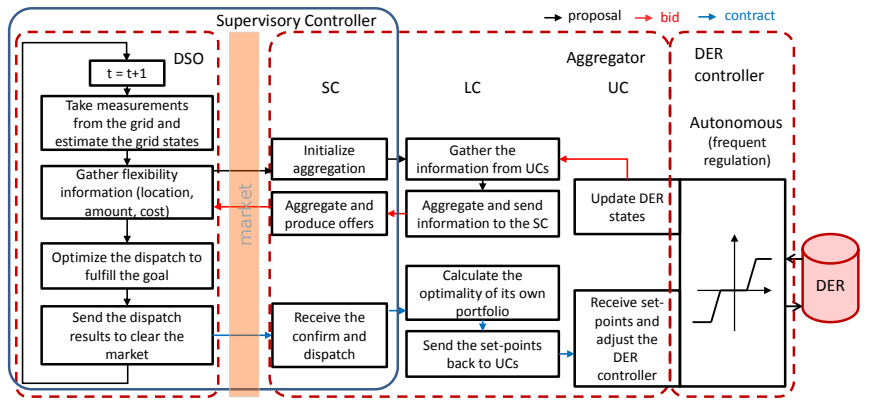

Fig. 3. The flowchart of the tailored hybrid control structure.

available flexibility and controllable devices to find the optimal operating point of the grid, while the aggregator optimizes its own DER portfolio by taking the decision from the DSO as the control input. The market ensures the most efficient resources are allocated (e.g., taking the cheapest offer from the flexibility bids). The aggregation approach makes sure that the request from the DSO can be allocated to individual DER units and executed to improve the grid operation as a whole.

There is one thing to be noted from Fig. 2 and Fig. 3: since the voltage is not a global state in the power system, a centralized market based approach requires more detailed location information of DER units to make a more accurate dispatch decision. However, the aggregation can no longer exist, if the location of all the DER units are forwarded to the SC. What is more, the SC need to process tons of data from individual DER units in order to make an optimal decision, which is hard to realize. In this paper, a hybrid control scheme is proposed: in the first level the location of DERs electrically close to each other is assumed the same one (i.e., "zones"), while we only keep the location information at the second aggregating level. The zones are determined based on the electrical location of secondary substations and the grid topology, so that the impact of the reduced location information can be minimized. Under an aggregation area (i.e., one "zone"), DER units are regulating their energy output according to both the set-points from the LCs and the local grid conditions autonomously. The hierarchical control structure makes sure that the available resources can be coordinated in such way that the overall operating performance is close to the global optimal point. The autonomous control at the bottom of the structure guarantees that the local constraints, which are not forwarded to the higher levels in the hierarchy, will still be respected. In the meanwhile, the fast variations can be tracked without very frequent communication with external entities. In our setup, the aggregation procedure runs every 10 minutes, while the parameters in the autonomous controller are adjusted every 1 minute.

\section{COORDINATION ALGORITHM}

\section{A. Autonomous controller for DER units}

Reactive power output from PVs and active power charging load from EVs are the sources of flexibility. PVs and EVs are equipped with autonomous controllers to determine their behaviours. Voltage information with receding horizon [8] is 


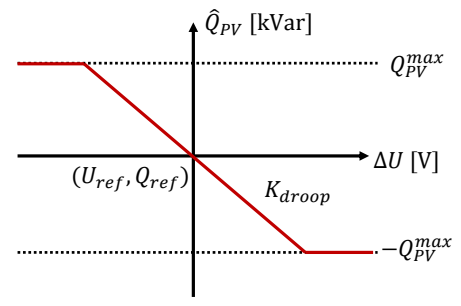

Fig. 4. Dependence of the setpoint on reactive power output.

used to capture the long term trend and short term variations:

$$
\begin{gathered}
\bar{U}_{n}(t)=(1-\alpha) \bar{U}_{n}(t-1)+\alpha U_{n}(t), \\
\widehat{U}_{n}(t)=(1-\beta) \widehat{U}_{n}(t-1)+\beta U_{n}(t), \\
\triangle U(t)=\widehat{U}_{n}(t)-\bar{U}_{n}(t) . \\
\sigma_{n}(t)^{2}=(1-\beta) \sigma_{n}(t-1)^{2}+\beta \triangle U(t)^{2}, \\
U_{d b}=K\left(U_{\max }-U_{\min }\right),
\end{gathered}
$$

where, $n$ is the node number in the feeder, $\bar{U}_{n}(t)$ is its voltage long term average, $\widehat{U}_{n}(t)$ is the moving average of a short interval, $\triangle U(t)$ is the long-term moving deviation, $\sigma_{n}(t)$ is the square root of the deviation, with $0<\beta \ll \alpha \leq 1$, and $U_{d b}$ is the dead-band of the voltage.

1) PV controller: Droop control is applied in the current setup as autonomous controller for PV inverters (see Fig. 4). Three parameters determine the control rules: reference point, dead-band, and slope of the droop. The reference point is the long term average voltage and the reactive power set point calculated from the hierarchical controller, if the aggregation is active. The slope of the droop $K_{\text {droop }}$ is calculated as

$$
K_{\text {droop }}=\frac{c_{P V}}{\sigma} \triangle U
$$

where, $c_{P V}$ is a proportional constant to scale the input signal.

2) EV controller: A varying threshold value for individual $\mathrm{EV}$ is used to determine whether one charges or waits. The willingness of the EV $i$ to start charging immediately is calculated as:

$$
w_{i}(t)=\frac{t_{\text {charge }}}{t_{\text {allowed }}} .
$$

where, $t_{\text {charge }}$ is the needed period to charge the battery to the accepted level, and $t_{\text {allowed }}$ is the period before the scheduled leaving time. $w_{i}(t)$ always lies between 0 and 1 . The threshold value $w_{i}^{t h}$ for $\mathrm{EV} i$ is determined by the following equation:

$$
w_{i}^{t h}(t)=\frac{c_{E V}}{\sigma P_{\text {available }}} \triangle U .
$$

where, $c_{E V}$ is the proportional constant, $P_{\text {available }}$ is the total rated charging power of available EVs connected to the zone. The EV will charge if $w_{i}(t)$ is larger than $w_{i}^{t h}(t)+\varepsilon . \varepsilon$ is a small random number that is used to diverse the charging behaviours of EVs to avoid critical kick-back effect [9]. If the hierarchical controller is enabled, the charging state may be changed by the set-point from the UCs.

\section{B. Hierarchical coordination}

The algorithm that is applied in the hierarchical controller is introduced in details in [6]. Here are some important functions:

- State Estimation In the current setup, power flow and voltage are measured in 6 nodes in the system (i.e., one node per zone) and is sent to the DSO. Furthermore, the load model and PV production is assumed to be updated given the historical data. The states of the grid are estimated based on such knowledge of the grid.

- Sensitivity Calculation Based on the grid state, the grid model is linearized at the operation point. The sensitivity between voltage and active / reactive power can be derived from such method.

- Optimization formulation The objective is formulated as a multi-objective function in a quadratic form. In this work, voltage deviation and cost of flexibility are considered and being minimized by taking 2-norm. The constraints are the maximal and minimal flexibility that is offered in each zone aggregated from UCs to the SC. The set-points of active power and reactive power usage are determined.

- Flexibility dispatch The set-points are dispatched to individual DER units by considering their current operation conditions and physical constraints (i.e., reflected in the cost formulation) in each level of the hierarchy from the SC to all UCs. The set-point for each unit is added onto the reference point of the droop curve. Therefore, the global goal can be respected by the whole DER portfolio.

\section{CASE STUDY}

\section{A. Grid and load model}

The distribution grid feeder is taken from a real Danish network [10] (see Fig. 5), which has a radial topology that connects to other feeders with normally opened breakers. The feeder contains 31 nodes, including 26 small customer groups and 14 large customers. The nodes are connected with each other through 31 underground cables. A secondary substation with step down transformer and lumped loads is connected at each node. Certain types of small customers are attached by flexible DER units, in our case, photovoltaic panels (PV) and electric vehicles (EV). The lumped load in the MV feeder under the last node is replaced by a LV feeder, which consists of 13 nodes (see Fig. 6), to tackle the problems on different voltage levels, and more specifically the voltage at the most critical point in the feeder (i.e., the far end of the feeder). The whole grid is separated into five zones, each of which is managed by a LC. All of these five zones are coordinated by a $\mathrm{SC}$ that is situated at the primary substation. According to the high penetration scenario in 2030 in Denmark [10], [11], EVs and PVs are connected into the grid model. The location and the population are associated with certain types of loads (e.g., residential houses and buildings), and their portions in the energy usage of the lumped loads. The secondary transformer is tapped with a fixed ratio of $1 / 1.025$. The average $\mathrm{R} / \mathrm{X}$ ratios of the cables in the MV and LV grid are approx. 2 and 3 respectively. The length of the MV feeder is approximately 


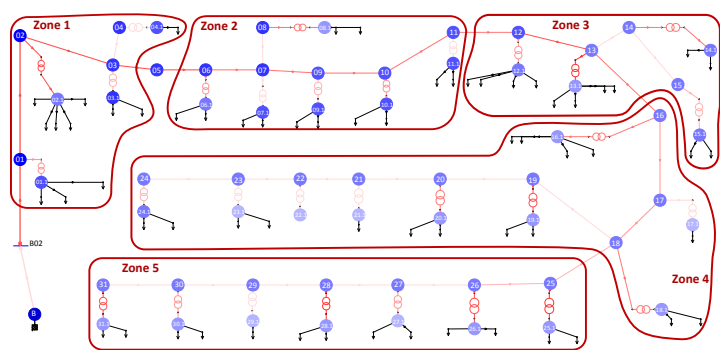

Fig. 5. A Danish MV feeder.

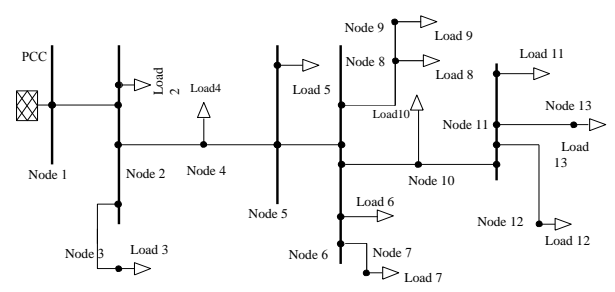

Fig. 6. A Danish LV feeder.

$20 \mathrm{~km}$, and it is $0.8 \mathrm{~km}$ for the LV part. The grid, loads, and DERs are modeled in MATLAB environment, and the simulation period is 5 days with 1 minute resolution.

\section{B. Scenarios and simulation results}

In order to evaluate the performance of the proposed method, three scenarios are constructed and simulated:

- S1: Without any control scheme. The PV panels do not inject reactive power, and the EVs charge when arriving at home.

- S2: Pure autonomous control scheme. The PV panels compensate reactive power and the EVs charge according to a fixed set-point.

- S3: Hybrid autonomous control scheme. The set-point for reactive power compensation and EV charging is determined by the hierarchical controller.

Fig. 7 shows the different portions of total active power consumption in the network with (S2) and without control (S1). It is seen that the load from EVs is shifted to midnight when the total load is comparably low. The results do not show any significant difference between the total consumption in S2 and S3, because the internal algorithm of the unit controllers tracks the variation of the voltage and adjusts the consumption accordingly. Whereas, less spikes are observed with the hierarchical coordination applied in S3.

Fig. 8 shows the behaviours of DER units with (S3) and without control (S1). In Fig. 8a, the green curve shows the reactive power compensation from an arbitrary PV panel in the network, and the blue curve is the active power injection. The figure shows the PV inverter compensates some reactive power into the grid, when the network load is large and the voltage is less than the average level, while it absorbs reactive power when the network load is little and the voltage is higher than the average level. The period when no reactive power is injected/absorbed by the inverter is when the voltage deviation is less than the allowance of the dead-band. Fig. 8b illustrates the charging behaviour of an arbitrary EV. The figure on the

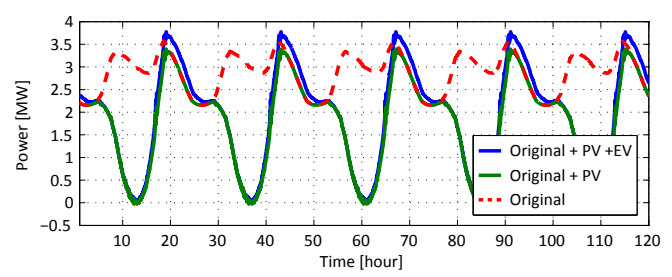

(a) $\mathrm{S} 1$

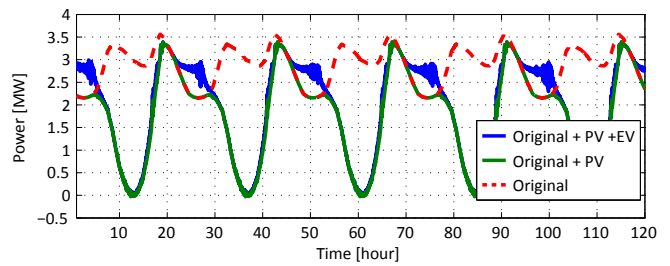

(b) $\mathrm{S} 2$

Fig. 7. Total power consumption profiles along time.

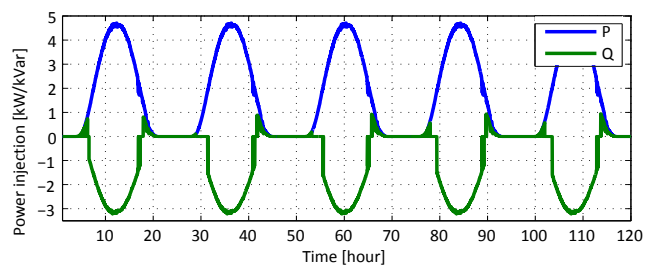

(a) PV output profile
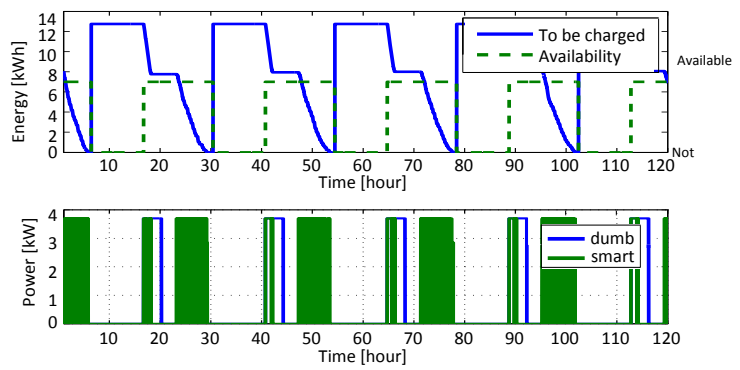

(b) EV charging profile

Fig. 8. Behaviours of individual DER units in the system.

top shows when the EV is available for charging and how the energy to be charged reduces along time. The daily driving consumption is added when the EV leaves home. The figure on the bottom shows that every night, when the load is large, the EV stops charging for a certain period, until the load is back to the normal range and the voltage increases to the acceptable value. The frequent variation of charging state is due to the fact that the charging decision is very sensitive to the level of energy remained to be charged, and it leads to smaller slope in the second night-charging period on energy reduction curve.

We also compare the flexibility usage of the DER fleets in different zones as presented in Fig. 9 given S2 and S3. It is observed that the average usage of the reactive power from Zone 5 is larger than the one from Zone 1 in S3. However, while it is almost the same in S2. In S2 the responsibility of mitigating the voltage deviation is the same for all the nodes in the grid, while it is determined by the hierarchical controller by considering their electrical locations in S3. From the optimization problem, it is identified that the change of 
power injection at the far end of the feeder could contribute the most. Therefore, the DER units in Zone 5 assume higher responsibility in absorbing reactive power from the grid to lower their node voltage.

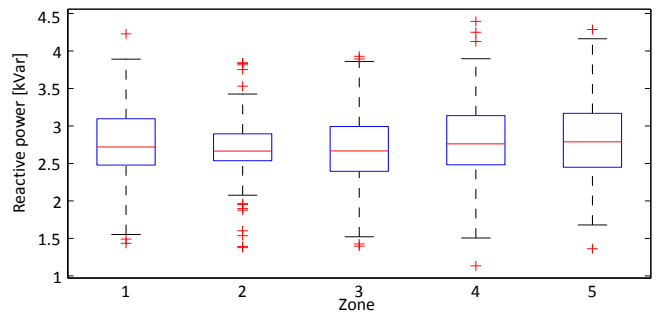

(a) $\mathrm{S} 2$

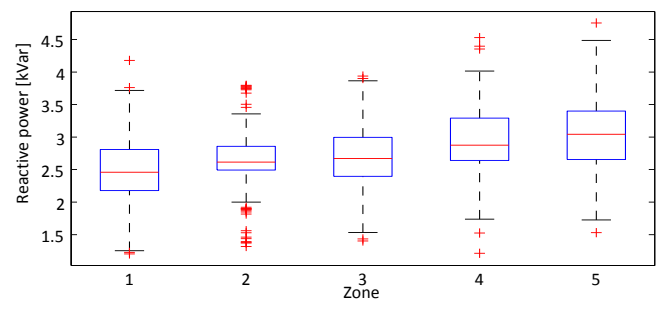

(b) $\mathrm{S} 3$

Fig. 9. Reactive power from PV inverters at 13:20 in different zones.

The cumulative distribution curves of voltage along time at the most critical point in the grid are plotted in Fig. 10 for all simulated scenarios. The high and low voltage situations are eliminated when the control algorithms are applied on the DER units. The lowest voltage magnitude in S2 and S3 is 0.915 p.u., while it is 0.9 p.u in S1. The highest voltage magnitude in S2 and S3 is 1.02 p.u., while it is 1.05 p.u. in S1. Under such peak and light load conditions, all the available flexibility is used to adjust the voltage profile. So the grid states are the same for S2 and S3. Comparing to the fully autonomous control, the hybrid control scheme provides better performance by better allocating the flexible resources along the distribution feeder. It is more often in S3 that the voltage is at $0.965-0.985$ p.u. than in $\mathrm{S} 2$.

\section{CONCLUSION}

In this paper, a hybrid hierarchical control scheme is proposed to enable flexibility services (i.e., in our work, voltage regulation) in a market environment. To overcome the barriers of a centralized approach for voltage services, autonomous control algorithms are embedded into the DER controller to respect both the request from the aggregator and

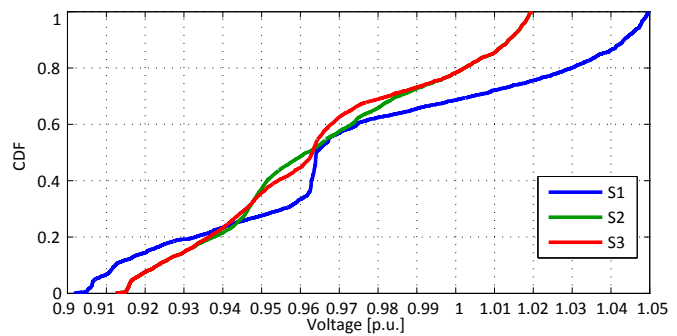

Fig. 10. Cumulative distribution of voltage at the far end of the feeder. the DSO. The performance of the proposed hybrid control scheme is evaluated and compared by simulating the grid performance with controlled DER units. The simulation results indicate that the control scheme could assist the grid operation by regulating the voltage in the distribution network. The emerging interconnection of DER units, such as PVs and EVs could provide strong potential for flexibility services.

From the simulation, there are a few interesting points that require more investigation:

- The zones in the work are predefined by comparing the amount of DER units, their electrical locations, and the electrical distances between nodes. An algorithm to draw the boundaries among zones is essential for the market to face the challenges brought by dynamic topology, and the large changes of DER locations (e.g., EVs may have certain patterns change their location for charging).

- The distribution of the state of charge of the EV batteries in the grid does not follow a typical distribution, and changes along time. Therefore, barriers exist on formulating the relation between the change of the voltage deviation and the change of the amount of EVs changing their charging states. Kick-back effects are observed in the simulation when an identical threshold value is assigned to a certain amount of EVs, and their willingness to charge is close to each other. The model of the dynamics of an EV fleet need to be improved to avoid such risks and to provide more accurate active power regulation.

\section{REFERENCES}

[1] The 2020 climate and energy package. European Commission, Last retrieved 20.10.2014. [Online]. Available: http://ec.europa.eu/clima/ policies/package/index_en.htm

[2] A. Keane, L. F. Ochoa, E. Vittal, C. J. Dent, and G. P. Harrison, "Enhanced utilization of voltage control resources with distributed generation," Power Systems, IEEE Transactions on, vol. 26, no. 1, pp. 252-260, 2011.

[3] M. Bayat, K. Sheshyekani, and A. Rezazadeh, "A unified framework for participation of responsive end-user devices in voltage and frequency control of the smart grid," Power Systems, IEEE Transactions on, 2014, early access.

[4] X. Han, A. Kosek, D. Bondy, H. Bindner, S. You, D. Tackie, J. Mehmedalic, and F. Thordarson, "Assessment of distribution grid voltage control strategies in view of deployment," in Proceedings of 2014 2nd IEEE International Workshop on Intelligent Energy Systems. IEEE, 2014.

[5] K. Heussen, D. E. M. Bondy, J. Hu, O. Gehrke, and L. H. Hansen, "A clearinghouse concept for distribution-level flexibility services," in Innovative Smart Grid Technologies Europe (ISGT EUROPE), 2013 4th IEEE/PES. IEEE, 2013.

[6] X. Han, A. M. Kosek, O. Gehrke, H. W. Bindner, and D. Kullmann, "Hierarchical control architecture to activate distributed energy resources' services: Voltage control as an application," in $T \& D$ Conference and Exposition, 2014 IEEE/PES, 2014.

[7] A. M. Kosek, O. Gehrke, and D. Kullmann, "Fault tolerant aggregation for power system services," in Intelligent Energy Systems (IWIES), 2013 IEEE International Workshop on. IEEE, 2013, pp. 107-112.

[8] P. Douglass, R. Garcia-Valle, J. Ø stergaard, and T. O., "Voltage sensitive load controllers for voltage regulation and increased load factor in distribution systems," Smart Grid, IEEE Transactions on, vol. 5, no. 5, pp. 2394-2401, 2014

[9] F. Sossan, X. Han, and H. Bindner, "Dynamic behaviour of a population of controlled-by-price demand side resources," in PES General Meeting-Conference \& Exposition, 2014 IEEE. IEEE, 2014, pp. 1-5.

[10] DONG Energy. (2014). [Online]. Available: http://www.dongenergy.dk/

[11] Energinet.dk. (2014). [Online]. Available: http://www.energinet.dk/ 